\title{
Research into practice: Collaboration for Leadership in Applied Health Research and Care (CLAHRC) for Nottinghamshire, Derbyshire, Lincolnshire (NDL)
}

\author{
Emma Rowley ${ }^{1 *}$, Richard Morriss ${ }^{2}$, Graeme Currie ${ }^{3}$ and Justine Schneider ${ }^{4}$
}

\begin{abstract}
Background: To address the problem of translation from research-based evidence to routine healthcare practice, the Collaboration for Leadership in Applied Health Research and Care for Nottinghamshire, Derbyshire, and Lincolnshire (CLAHRC-NDL) was funded by the National Institute for Health Research as one of nine CLAHRCs across England. This paper outlines the underlying theory and its application that CLAHRC-NDL has adopted, as a case example that might be generalised to practice outside the CLAHRC, in comparison to alternative models of implementation.

Discussion: Conventional approaches to health research frequently generate evidence in isolation from the environment in which it is intended for use. The premise of the CLAHRC-NDL model is that barriers to implementation can be overcome if knowledge is co-produced by academic and clinical service staff, taking account of the organisational context in which it is to be applied. This approach is founded on organisational learning theory, recognising that change is a social and political phenomenon. Evidence is produced in real time, taking full account of the environment in which it is to be implemented. To support this process, senior health service staff are seconded to the CLAHRC as 'diffusion fellows' (DFs) to actively bridge the research to practice gap by being a full member of both the research team and their area of clinical practice. To facilitate innovation and embed change in the local health community, existing communities of practice are enhanced and new ones are fostered around specific themes. Our approach has been adopted by 16 clinical research studies in the areas of mental health, children and young people, primary care, and stroke rehabilitation.

Summary: The CLAHRC-NDL model of implementation applies organisational learning theory by addressing the social and situational barriers and enablers to implementation, and adopting a philosophy of co-production. Two key mechanisms for translation of innovation have been utilised: DFs, to actively bridge the research to practice gap, and communities of practice, to underpin and sustain improvements in healthcare. The model shows promising results in putting research into practice, which may be transferable to other healthcare contexts.
\end{abstract}

Keywords: CLAHRC, Research into practice, Implementation, Knowledge mobilisation, Organisational learning, Translation, Communities of practice

\footnotetext{
* Correspondence: Emma.Rowley@nottingham.ac.uk

${ }^{1}$ CLAHRC-NDL/Nottingham University Business School,

Institute of Mental Health, University of Nottingham Innovation Park,

Nottingham NG7 2TU, UK

Full list of author information is available at the end of the article
} 


\section{Background}

Research evidence does not easily or predictably translate into patient care [1,2]. Rycroft-Malone et al. [3] assert that 'the rational-logical notion that producing research, packaging it in the form of guidelines and assuming it will be automatically used is now outdated.' The Medical Research Council (MRC) framework on complex interventions [4] recognises that 'passive (implementation) strategies are ineffective in getting evidence into practice.' Research and practice are unaligned, leading to poor health outcomes, inequalities, and wasted resources [5].

Dearing [6] describes a 'quality chasm,' whereby 'the state of science (what researchers collectively know) and the state of the art (what practitioners collectively do) coexist more or less autonomously, each realm of activity having little effect on the other.' Similarly, the Chief Medical Officer's High Level Group on Clinical Effectiveness reports on the need for the National Health Service (NHS) to better utilise the skills and knowledge of academic colleagues, in order to improve efficiency in delivering clinical care [7].

\section{CLAHRCs as a solution?}

In order to address these shortcomings, the UK's National Institute for Health Research (NIHR) funded nine applied research units across England (2008 to 2013). Collaborations for Leadership in Applied Health Research and Care (CLAHRC) were established to address problems with the translation of research into practice-the 'second' translation gap [8] or the 'know-do gap' [9].

Kislov et al. describe the CLAHRC initiative as a 'major experiment ... with an ambitious goal of creating a new, distributed model for the conduct and application of applied health research that links producers and users of research' [10]. This paper discusses the approach taken by CLAHRC Nottinghamshire, Derbyshire, and Lincolnshire (NDL) in closing the research to practice gap. Our model is founded on the premise that evidence is inevitably applied in a particular context. This means that interventions fail because they are not fit for purpose and/or have no organisational alignment.

We suggest that our approach offers the possibility for more nuanced and contextually relevant insights that more structural implementation frameworks may overlook.

\section{Discussions}

The interaction between research and practice is frequently characterised by poor communication and cultural differences [11-13]. Academic research outputs often speak a different language and address a different set of objectives from those pursued by clinicians. This provides one reason why research evidence is not implemented into clinical practice.

Investigators have long recognised that knowledge mobilisation is not a simple linear process, but occurs through social systems whose complex interactions may facilitate, transform, or obstruct the transfer in unanticipated ways with unexpected consequences. Hence the term 'knowledge mobilisation' has increasingly supplanted the more linear notion of 'knowledge transfer' [14]. We use the term 'knowledge mobilisation' to describe the translation of evidence into practice, and recognise it as a central part of the dissemination and implementation process which aims to close the research to practice gap.

However, less is known about how knowledge mobilisation, specifically the translation of evidence, can be designed into research studies. Traditionally, research was carried out, and then a separate team adopted an implementation strategy to put the evidence generated into practice $[7,15]$. For example, usual practice may have been to wait for a stimulus, such as a National Institute for Health and Clinical Excellence (NICE) Guideline or health technology appraisal, a commissioning process, or public or professional pressures to adopt an intervention, before working towards implementation. However, the linear and haphazard process of evidence production followed by implementation is not only inefficient, but also ineffective in closing the research to practice gap and thereby improving patient care.

\section{Bridging the gap}

Eccles et al. [16] suggest that implementation research is an 'expensive version of trial and error', and the coherency of its theoretical basis has been challenged [3]. However, Wilson et al. [17] identify 'several theories and frameworks' that can be used to guide or explain the translation of evidence-based programmes, practices, and policies into practice:

1. Knowledge to action models (KTA) separate the production of knowledge from its application. Numerous KTA models exist, making it 'difficult for researchers and managers to choose which model to use' [5]. Ward et al. [5] identify five common components: problem identification and communication; knowledge/research development and selection; analysis of context; knowledge transfer activities or interventions; and knowledge/research utilisation.

2. The Quality Enhancement Research Initiative (QUERI), enhances healthcare through the production of research-driven initiatives [18]. It sees implementation in terms of cultural norms and values, capacity and supportive infrastructures. QUERI employs a six-stage process, from identification of problem and best practice, identification and implementation of new interventions, through to an evaluation of whether the intervention has improved outcomes. 
3. Normalisation Process Theory (NPT) [19-21] provides a framework to consider the social processes relating to how new innovations become embedded in everyday practice. It also aims to assess the probability of the intervention becoming implemented.

4. Promoting Action on Research Implementation in Health Services (PARIHS) considers the interplaying factors that influence or lead to successful implementation (SI), including the function of the nature of the evidence (f), the type of evidence (E), the context $(\mathrm{C})$ and the process of facilitation $(\mathrm{F})$ [22]. It does this by following a formulae for successful implementation: $\mathrm{SI}=\mathrm{f}(\mathrm{E}, \mathrm{C}, \mathrm{F})$.

5. The Plan-Do-Study-Act (PDSA) cycle is a linear approach to translation. It comprises of four distinct stages: plan the change to be tested or implemented; carry out the test or change; study the data before and after the change to reflect on what was learned; and act-plan the next change cycle or full implementation [23].

While each of these frameworks has been used successfully, they do not entirely fit with the epistemological position taken by CLAHRC-NDL. Our approach recognises that 'the different players within healthcare-clinicians, epidemiologists, health services researchers, educationalists, social scientists, economists, health authorities-often have different ideas on the best strategies to improve practice and the best way of making changes' [24]. The aforementioned models are relatively linear, inclined towards individual-level behaviour change, with less recognition of the need for organisational-level change. Moreover, the models are largely silent about the epistemological challenges of mobilising knowledge, which include the nature of knowledge or evidence, and associated political and cultural dimensions of knowledge mobilisation.

\section{The CLAHRC-NDL approach}

CLAHRC-NDL is based on organisational learning theory, an approach that generates knowledge directly within the area of practice it is to be implemented. This approach is distinctive in combining elements from social sciences and health services research with academic and non-academic clinical expertise, organisational leadership and service user and carer perspectives [25-27]. From their organisational learning perspective, EasterbySmith et al. [27] highlight a practice-based view of knowledge translation. Knowledge is acquired tacitly, and its translation influenced by political and cultural considerations. These challenges are known to be particularly pronounced in healthcare settings $[25,26]$. However, we moved beyond the organisational studies literature from which the idea of organisational learning emanates, to consider the healthcare professional and patient and carer perspective on knowledge mobilisation in more detail. This involved drawing on health services research about knowledge mobilisation [15,28-33] and sociology of health and illness literature about patient and carer involvement in evidence-based practice [34-38].

The Tooke Report [7] supports our theoretical position on the need for organisational learning to mediate potential blockages to implementation, highlighting how systematic, multi-layer interventions are required to translate evidence-based healthcare into routine clinical practice, in order to meet local and national priorities. Ferlie and Shortell [39] argue that implementation requires 'explicit consideration of the multilevel approach to change that includes the individual, group/team, organisation, and larger environment/system level.' This perspective additionally takes account of organisational receptiveness to the intervention, and to developing the capacity of healthcare staff to reflect upon and evaluate the implementation of innovation. Organisational learning is rooted in the social $[27,40]$; it helps us to understand implementation (translation) as an enacted and situated phenomenon [41,42]. The CLAHRC-NDL approach therefore encourages a reflective understanding of 'what is going on' [40].

We regard change as a social and political phenomenon [25], rather than one where the knowledge base for change is decoupled from practice. Knowledge production and translation therefore requires 'situated' learning [43]. Such a need relates not only to political and cultural dimensions of implementation, but the nature of knowledge itself. For example, clinical academics and practitioners may be unwilling to exchange knowledge, where this would run counter to professional self-interest. Alternatively, they may be unable to exchange knowledge, since their perspectives on healthcare problems and solutions may diverge, as evidenced in different professional discourses.

Knowledge is not just formal evidence produced through randomized controlled trials (RCTs), which becomes codified into national guidelines; knowledge is often tacit, and embedded in practice. It is rarely articulated and codified, and therefore it is seldom open to challenge and modification. Our approach makes this tacit knowledge more explicit to practitioners themselves and those who work alongside them, so that the evidence base for change is linked to clinical practice in real time, as problems arise and as solutions are found [26]. In this way, the development and use of evidence is intertwined and takes account of the organisational context in which implementation takes place. Not only is evidence embedded in practice, but it is also co-produced by researchers, practitioners, service users, and managers. Such a process requires a culture of trust and 'give and take' by all parties, including clinicians and academics who may be used to holding sway over their respective domains. The iterative co-production 
of knowledge contrasts with more traditional, linear models of knowledge transfer. Instead of context being controlled for, as with experimental RCT-based design $[44,45]$, taking a situated learning approach requires that context is built into the research design so that local learning and change is engendered. Thus, organisational learning research can be used in a formative manner to introduce and incrementally improve a new intervention. It can also be used to improve standard care if an intervention ultimately proves to be unfeasible, ineffective or too costly.

The organisational learning approach to implementation is about behavioural change, but it goes beyond the individual level of analysis (although this is still necessary to understand organisational and institutional barriers and facilitators to change). It demands that research be sensitive to local context(s) in order to understand how something works in everyday practice (referred to as 'the active ingredients' [46]). This can include exploring the 'how,' 'when', 'why', and 'where' factors that help to explain the successful or unsuccessful implementation of an innovation.

\section{Co-production of knowledge as part of situated learning}

The model is designed to highlight political and cultural barriers to change, develop an understanding of the barriers and drivers to translation of evidence into practice, and to recommend prescriptions to overcome these. The emphasis of CLAHRC-NDL lies in a situated model of translation concerned with developing community tendencies towards the implementation of change, which can arguably overcome political, cultural, and structural barriers to the translation of innovation into practice. This is important, because healthcare is delivered by multi-disciplinary and multi-organisational networks, with the consequence that a critical mass of stakeholders, rather than a single clinical champion, is necessary to mobilise knowledge in pursuit of changing clinical practice $[28,29,47]$.

The co-production of knowledge requires give and take to meet the needs of both academic and clinical staff so that the research project is fitted to the healthcare organisation environment. This might be in terms of what interventions are studied, how they are delivered, or what outcomes and processes are evaluated. Concessions towards academic rigour are made, such as through the randomisation and completeness of data collection. Central to the organisational learning approach is an understanding of the advantages of such co-production to all involved: ideally, academic staff gain by attracting greater numbers of participants, wider understanding of the research problem and insights that ultimately lead to improved interventions, and therefore opportunities for further research; clinicians and service users gain by updating their practice and collecting evidence of impact, rather than anecdote and audit; managers and commissioners gain by knowing that cutting-edge practice is being applied quickly and systematically within local health services. Thus, by the end of the research process, clinical practice is merely a refinement of the service development, and its evaluation is carried out during the research itself, making a second separate stage of implementation of knowledge from research into practice largely redundant.

\section{Broadening and sustaining the impact of change}

The organisational learning model captures how research can be adapted and become fit for purpose in the clinical area of practice where it might have the greatest impact. The needs of clinicians, service users, and managers who are enthusiasts, innovators, or early adopters of change $[19,48]$ will have been met by such an approach. However, staff leave, new imperatives emerge, and knowledge can be lost in the flux of organisational change, incoming clinical staff may be more reticent or less able to directly engage in the co-production of the research with academics. Embedding quality improvements in the culture of a health community calls for a broad consensus, with its own impetus and leadership, which is relatively impervious to the vicissitudes of health policy and politics. We approached this by engaging, training, and supporting researchers, health and social care professionals, patients, and public representatives to develop a community of practice (CoP) around each co-produced research study [49]. The primary function of each $\mathrm{CoP}$ is to sustain change and drive forward innovation. Although more distant to the process, CoP members are encouraged to become involved in how the clinical innovation is implemented. For instance, they may wish to clarify areas of uncertainty and highlight improvements in how the innovation is presented and delivered. Such perspectives can be influential and enable us to gain deeper, richer, and more explicit understandings of the innovation as it is exposed to a greater range of clinical challenges over time. In this sense, the principle of coproduction is maintained, leading to a sense of ownership by the $\mathrm{CoP}$ of the change being introduced, thereby addressing some of the cultural and political barriers to its implementation. Consequently, if it is managed well, the coproduction of innovation has the potential to ultimately change both the culture of the CoP [50] and to play a significant role in the wider healthcare organisation, so that both are more receptive to other co-produced innovations, and the process of evaluation and research.

Embedded within each of the 16 studies is our implementation work, which consists of two distinct programmes. The implementation theme, distinctive from the clinical themes, poses implementation as both a research problem and as a practical problem in education and change. Staff from the implementation theme took a lead in training and supporting individuals seconded into 
the CLAHRC from the NHS as knowledge brokers, so they could work effectively alongside their academic partners, and also develop a broader understanding of commissioning and service provision needs of local NHS and social care organisations to understand the full relevance and potential of the project work. In addition, researchers from the implementation theme take a lead role in carrying out the qualitative, exploratory work to understand the multilevel approach to change that Ferlie and Shortell [39] outline. This includes exploring the barriers and facilitators to change (knowledge mobilisation and implementation) at individual, group/team, organisation, and institutional levels.

\section{Putting the CLAHRC-NDL approach into practice}

NIHR CLAHRC-NDL covers the northern part of the East Midlands of England, an area of diverse health and social care need. It has a population of approximately three million, and a varied geography, encompassing deprived city centres, urban conurbations, and rural areas. Like all CLAHRCs, our funding was awarded by the NIHR and a combination of academic and practice partners. CLAHRC-NDL has four research themes covering primary care, mental health, children and young people, and stroke rehabilitation. These themes are supported by two implementation themes, implementation, and engagement, synthesis and dissemination (see Figure 1). Both of these themes work into the clinical studies to ensure that the research is aware of the implementation and engagement issues.

The primary aims of the four research themes are:

1. To study issues identified as local priorities and feasible to change, and to improve clinical care to at least the best current national standard.

2. To explore whether organisational learning research is feasible, informative and of additional use to the process of commissioning and service delivery of NHS services.

3. To develop capacity in the NHS workforce so that commissioners and providers can undertake organisational learning research with minimal academic supervision.

Figure 2 shows how the organisational learning approach is operationalised. In May 2008, a survey of commissioners, clinicians and users in the East Midlands was carried out to determine local interest and relevance of our proposed studies and theoretical approach. Studies were chosen to address issues of local importance (as defined by stakeholders) and determined by local policy documents $([11,12,51]$, and NHS and local authority local commissioning plans and priorities), whilst also reflecting the NIHR's call for CLAHRCs to concentrate upon public health and chronic disease. Scoping interviews were carried out with heads of specialist services, commissioners, clinicians and service users in NHS and local authority organisations across our region. In all, 26 of 34 (76\%) participants responded; CLAHRC-NDL's aims, objectives, and proposed project outlines were discussed, and feedback given. This resulted in some amendments to the scope of some studies, whilst others were not taken forward.

The relevance of each project to the NHS, social care, commissioning, and service provider agenda is maintained at a later stage of protocol development by its consideration at a commissioner's panel consisting of representatives from each of the partner organisations. To ensure that the projects benefit from service user and carer experience, each study is reviewed by a service user and carer panel, and service users are part of each study's steering committee. The scientific methodology of each project is reviewed and monitored annually by an externally appointed scientific committee consisting of academic experts in health policy and management, implementation, medical statistics, and health economics supplemented by peer subject review from external clinical academics. The scientific committee represents a crucial mechanism to ensure clinical academic research projects continue to prioritise implementation in an active way. Our 16 studies are shown in Table 1.

The designs of our empirical studies are varied. Typically, interventions at an early stage of development

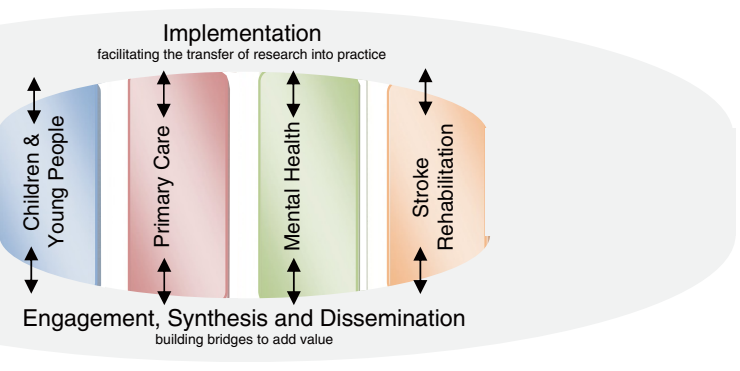

Figure 1 CLAHRC-NDL Themes. 


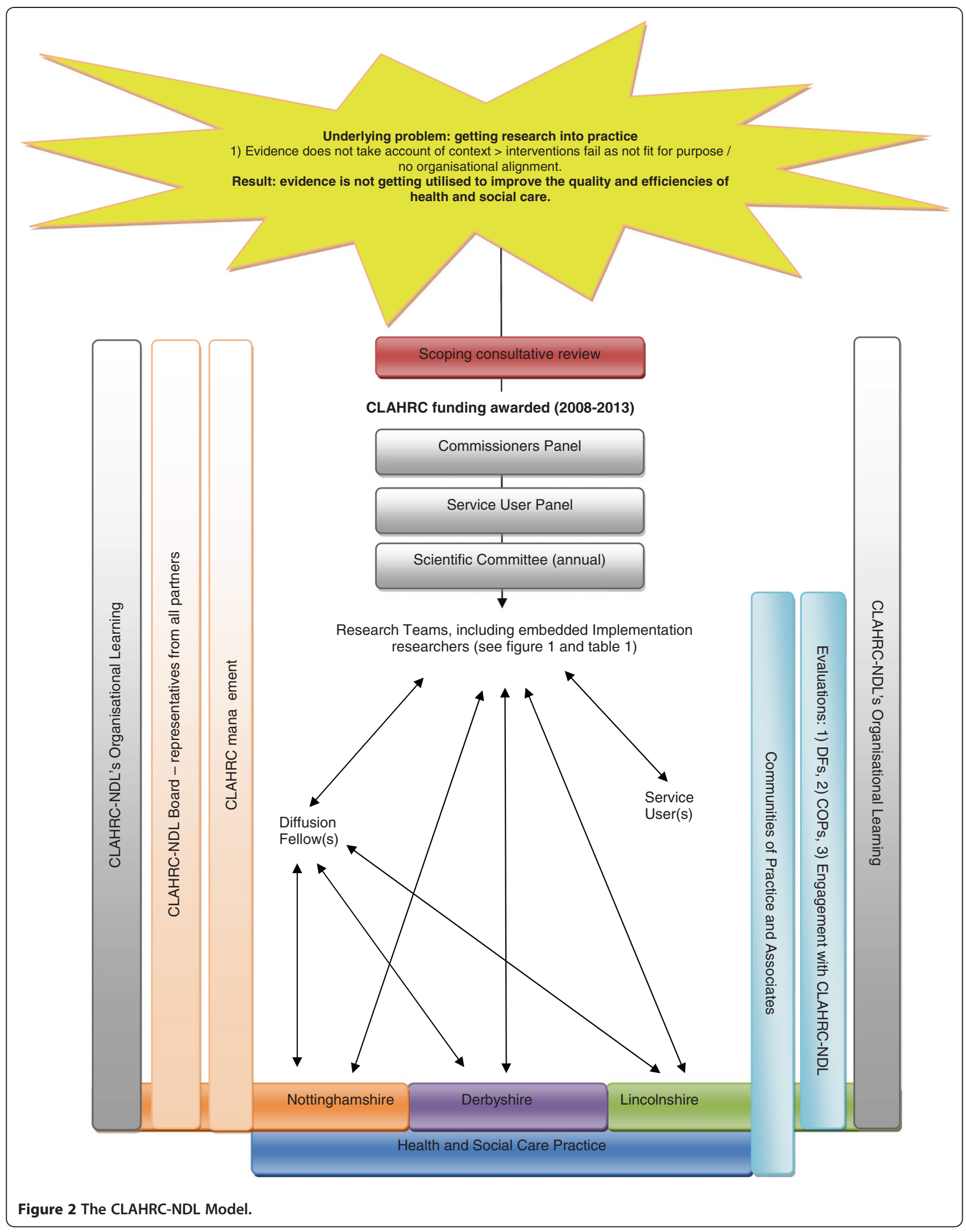


Table 1 CLAHRC-NDL studies by theme ${ }^{1}$

\begin{tabular}{|c|c|c|c|}
\hline Mental Health & Primary Care & Stroke Rehabilitation & Children and Young People \\
\hline $\begin{array}{l}\text { Readiness for treatment } \\
\text { for people with } \\
\text { personality disorder. }\end{array}$ & $\begin{array}{l}\text { Reducing the risk of diabetes } \\
\text { amongst members of the } \\
\text { South Asian population in } \\
\text { the East Midlands. }\end{array}$ & Return to work after Stroke. & $\begin{array}{l}\text { Consensus in assessment and } \\
\text { treatment outcomes for children/ } \\
\text { young people's mental health } \\
\text { (CATO). }\end{array}$ \\
\hline DFs: 2 & DFs: 1 & DFs: 1 & DFs: 1 \\
\hline $\begin{array}{l}\text { Dental and physical } \\
\text { health needs of people } \\
\text { with serious mental } \\
\text { health problems. }\end{array}$ & Exploring preconception care. & Home visits after Stroke. & $\begin{array}{l}\text { Medication optimisation strategy } \\
\text { for ADHD management in } \\
\text { childhood (MOSAIC). }\end{array}$ \\
\hline DFs: 1 & DFs: 1 & DFs: 1 & DFs: 2 \\
\hline $\begin{array}{l}\text { Supported employment } \\
\text { for people with severe } \\
\text { mental health problems. }\end{array}$ & The impact of injuries study. & $\begin{array}{l}\text { Home-based upper limb virtual } \\
\text { reality rehabilitation after Stroke. }\end{array}$ & $\begin{array}{l}\text { Group parenting programme, } \\
\text { for children with ADHD } \\
\text { (PATCHWORK). }\end{array}$ \\
\hline DFs: 1 & DFs: 2 & DFs: 1 & DFs: position currently vacant. \\
\hline $\begin{array}{l}\text { Trial of cognitive behavioural } \\
\text { therapy for people with } \\
\text { chronic mood disorder. }\end{array}$ & $\begin{array}{l}\text { Regular attenders with } \\
\text { medically unexplained symptoms. }\end{array}$ & $\begin{array}{l}\text { Implementation of Early Supported } \\
\text { Discharge following Stroke. }\end{array}$ & $\begin{array}{l}\text { Steps to Active Kids-a } \\
\text { school-based activity programme } \\
\text { (STAK). }\end{array}$ \\
\hline DFs: 2 & DFs: 1 & DFs: 1 & DFs: 2 \\
\hline
\end{tabular}

${ }^{1}$ There is a CoP for each of the 16 CLAHRC studies although Associates may belong to more than CoP in a theme.

employ a scoping or case study design, while more developed interventions employ feasibility RCT design. In the case of studies examining the identification of the need for care, mixed methods research is used. Our overarching implementation research programme is a proof of concept exploring how feasible and useful the organisational learning approach is to the NHS, and whether it is effective in helping to close the research to practice gap. Consequently, interventions have been designed according to the principles laid out in the MRC Complex Interventions Framework $[4,46]$.

\section{Situated learning}

The situated learning in CLAHRC-NDL is primarily achieved through the secondment of 24 individuals from our partner organisations, called diffusion fellows (DFs). We note the discrepancy between organisational learning and the field of knowledge brokering here, however the name 'diffusion fellow' was most attractive to our practice partners. In the spirit of co-production we adopted the name, yet as academics informed by institutional theory, we recognise the distinction between diffusion and translation, as one where the latter has more emphasis upon the way evidence is modified and acted upon by stakeholders as it moves into frontline healthcare practice [52]. DFs act as change agents, and champions for innovation, influencing both the production of knowledge and also its mobilisation. In enacting this hybrid role, DFs help our research teams to understand and appreciate the implementation context, prompt modifications to the design of the intervention (to aid later implementation), and also take the knowledge gained about the research back into their organisations and practice-based networks.

DFs are aligned to each of the 16 clinical research projects (studies have one to three DFs each). They are senior clinical or managerial staff (consultant doctors, matrons, allied health professionals, and senior directorate managers), appointed by the partner organisation because of their interest in the project and their position to influence change within the organisation. DFs are seconded to the project for one day per week, for up to five years, with their secondment funded as part of the matched funding received from partner organisations. Their purpose is to optimise the research so that it best fits the needs of the NHS without compromising academic rigour; to publicise the research; to promote recruitment; and to implement better clinical and managerial practice as findings emerge from the projects. Over time some DFs have played a central role in creating, developing and maintaining the CoPs within CLAHRCNDL. The role of DFs is being evaluated [53].

The engagement, synthesis and dissemination initiated the development of CoPs (engagement), working across research projects to derive added value (synthesis), and promoting the publication of CLAHRC-NDL outputs in relevant forms (dissemination). The CoPs model was adopted to provide a meeting ground focused around research areas [50] building on existing and facilitating new networks of health and research-oriented members within the CLAHRC. Although in Table 1 there seems to be an overlap between projects in terms of the clinical condition, 16 CoPs were effectively formed as each project tackled implementation and knowledge mobilisation in a particular settings, thereby engaging different networks. Stakeholders are recruited as 'associates' affiliated 
to specific CoPs, each linked to a project or theme, to focus their involvement with the CLAHRC. The crosscutting activities of the engagement theme were planned to promote and encourage CoPs, based upon the four characteristic activities identified by Li et al. in their systematic review [36] — social interaction among members, knowledge sharing, knowledge creation and identity building, all directed at creating a self-sustaining network that is receptive to organisational learning in relation to the research areas. A database was set up to facilitate the links between the CoPs and associates, and within the first year, 400 people had joined as associates linked to one of the research themes.

There was a pre-existing culture of CoPs in Nottinghamshire; the Institute of Mental Health offers small grants to 'managed innovation networks' made up of clinicians, service users, and researchers working on a common purpose. In addition, there is an established stroke survivors group that serves a similar purpose. The additional resources of the CLAHRC helped existing CoPs to reinforce their identity, by holding regional meetings, extending their networks, attending national conferences, producing newsletters, and organising stakeholder events. No existing CoPs were identified in child and adolescent mental health nor in primary care, so new networks were fostered through engaging with researchers and DFs, encouraging them to identify others with shared interests, to recruit them as associates and to involve these people in CLAHRC-related activities, such as the annual conference, local roadshows, monthly updates, regular newsletters, and event postings. CoPs are diverse and are actively contributing to the CLAHRC enterprise.

The CoPs set their own ground rules for meeting, although they continue to use both project and CLAHRC corporate based communications. Face to face meetings are often task related. For example, in the chronic mood disorder study, the CoP consisting of therapists, researchers, and service users identified the need for follow on groups after psychological treatment for depression that could be run by service users. It also generated insights to the evaluation of this study through the members' observations of how engagement of service users in psychological treatment had improved compared to usual care [54]. Another example, the return to work after stroke study, has successfully engaged service users, carers, and employment support providers from outside the health arena in a CoP that also includes occupational therapists and researchers in stroke rehabilitation. They have identified a pro forma for capturing the content of the intervention and helped to identify and refine key components to its success.

The aim of the CoP initiative is to enable these networks to become 'self-sustaining' within the lifetime of the CLAHRC. The eventual devolution of responsibility for leading the CoPs to their membership is anticipated; this is essential for their continuity, given the timelimited funding of the CLAHRC.

\section{Translation and communities}

While our organisational learning approach helps to consider the situated implementation issues that are pertinent to each study, additional assistance is needed to mobilise the knowledge and evidence produced, and to get the research implemented. Change is issue-centered, homegrown, and collectively implemented [34]. Therefore having a contextual understanding of the organisation or service into which change is to be actioned and implemented is critical. However, as 'outsiders', academics are not often granted full access to this.

The DF model is a unique element of our attempts to get research into practice through situated learning in real time. This is reinforced by the iterative process of knowledge mobilisation between project teams and clinical services (at various levels, such as DFs, CLAHRC associates, commissioning panel, and service user group, as illustrated in Figure 2) rather than the more passive transfer of knowledge from academic research to clinical practice that is traditionally attempted through clinical guidelines, local and national policy, and standards set by professional bodies such as Royal Colleges.

The manner in which DFs work with their research teams is individually negotiated. DFs work inwards to the research project, advising on the design of the study, and then throughout the project in relation to identifying and solving practice-based 'real world' issues and practicalities. Moreover, together with the research teams, they also work outwards, from the research base into practice, disseminating the evidence into the stakeholder communities, and working towards the commissioning of new services based on the evidence produced. DFs contextualise and help to translate the research, so that the evidence produced is relevant to those commissioning care or applying it in their own practice. In doing this, we rely very much on their tacit, rather than explicit knowledge, which assists in accelerating the mobilisation of evidence-based innovation into practice. This will build the capacity of the DFs to tackle and implement change in their own organisations. In turn, our health and social care partners will become more receptive to evidence, and our academics will consider the context in which their work is to be applied, thus closing the research into practice gap.

Lave and Wenger [43] describe how individuals support situated learning through their participation in CoPs. This extended reach is of vast value to the work of the CLAHRC, as it enables knowledge mobilisation to engage a wide(r) swathe of stakeholders involved in evidence implementation and evidence based practice (see Figure 2). This means that knowledge mobilisation, as a collective phenomenon, goes beyond the individual clinical 
champion; for example, DFs bring added value to our knowledge mobilisation work through access to their networks/CoPs. Following Tsoukas and Vladimirou [55], we see knowledge mobilisation as situated action, and as an iterative process [56,57] involving horizontal processes, drawing on spheres of influence incorporating networks of peers and reframing and adapting knowledge by drawing on everyday practice, vertical processes relying on topdown information cascades from executive management or research bodies, and feedback processes following reflection on changes to practice as they start to be implemented. CoPs have an important function in relation to closing the research to practice gap. They are a means of translating knowledge and information into a network of interested parties, and can be formed along institutional, organisational, or professional affiliations, or can be based on a topic of interest. For our knowledge mobilisation purposes, CoPs are valuable in relation to their ability to foster situated learning [43], through members' shared common purpose or activity, which in turn can have knock-on effects in the implementation site; this is because the members of the CoP have a fundamental role in shaping the evidence, and the translating it back into their sphere (s) of influence [42]. It is this sense of shared practice and understanding amongst members of the CoP which enables knowledge mobilisation to occur smoothly [40].

\section{Evaluating this approach}

CLAHRC-NDL is performing its own internal evaluation, including an exploration of the role of DFs [53], CoPs, and the influence of the CLAHRC on other clinicians and managers. These studies will be detailed in separate publications. Externally, we have commissioned independent economic analysis of wider effects of the CLAHRC. Together, the evaluation work provide a comprehensive view of the added benefit, if any, that the application of organisational learning might bring to CLAHRC-NDL and to our health and social care partners.

Eccles et al. [1] argue that theoretical implementation research is advantageous as it 'offers a generalisable framework that can apply across different settings and individuals.' Our model emphasises the influence of social context and the notion of something evolving and acting 'in-practice' (see [58]). However, it also provides a set of general principles or learning points regarding how to carry out implementation research in real-world settings. In this way, the CLAHRC-NDL model resonates with the NPT framework [19-21], in that it identifies and describes factors that promote or inhibit implementation. However, unlike the NPT framework, our approach specifically caters for the complexity, fluidity and messiness that occurs in any social space.

The CLAHRC-NDL model is a middle ground between highly theoretical, structured implementation frameworks, and Oxman et al.'s [59] common sense approach founded on practical judgement. We practice what Eccles et al. [16] refer to as 'theory-based factors' or 'theoretical constructs' that are used to guide the development and implementation of interventions. This approach is both theoretically valid, allowing for 'rigorous' academic research, whilst also proving to be 'clinician' friendly and reflecting the diverse mechanisms, influences, and actors involved in the implementation process.

Another advantage of our approach over the various alternative models is its simplicity. Both the research and the clinical work (with some allied activity such as education or audit) would go ahead anyway without the involvement of the CLAHRC. However, clinical and research teams are channelled into these co-production activities. Some of the alternative implementation models require a considerable investment in infrastructure and are labourintensive; they involve additional posts and activities that are not usually performed. Our approach is arguably more resource-efficient than some of the alternatives outlined earlier in the paper.

Our model has limitations. Collaboration does not just happen; it takes effort and continual and dedicated management. For example, divides between education and the NHS are well known [60], siloed working barriers to collaboration across academic disciplines have needed negotiation. We were not successful in engaging all the acute Trusts across our geographical patch, the NHS reorganisation has altered our audience and led to the loss of key relationships, whilst the already-low capacity of local authorities to work with and utilise research has been further reduced by funding cuts resulting in a disappointing lack of engagement. There needs to be interest in the topic at both clinical and academic levels, as well as incentives to participate for all concerned. These incentives do not necessarily have to be financial and are often mostly driven by interest in the topic itself. Yet, to justify the time and commitment of those involved, there need to be tangible outcomes that are valued alike by the clinicians, researchers and their employing organisations.

We do not pretend that the implementation of every innovation could or should be tackled through organisational learning. Instead, we set out to test and challenge the feasibility and utility of it in a broad range of clinical conditions, and health and social care organisations. In addition, the approach is being tested in a period of unprecedented and unanticipated reorganisation and financial constraint in the publicly funded health service in England.

Moreover, our organisational learning approach is not suitable in establishing basic scientific principles, such as the efficacy and safety of relatively simple interventions, but would be suitable when simple interventions are combined and applied to broad clinical practice. There are also many methodological challenges to address such 
as conducting multi-centre studies using organisational learning involving clinical services and academic staff across many sites. Such multi-centre studies may be the only feasible way of obtaining an adequate sample for relatively rare conditions, and to test the generalisability of an approach outside one specific health setting.

\section{Summary}

The CLAHRC-NDL approach to closing the research to practice gap incorporates a multi-level interactive stance. It is situated and co-produced, taking account of the intricacies and needs of the provider organisations and the health and social care communities that we serve. Our knowledge mobilisation strategy-a key component of any attempt to implement research-is built around our DFs, and real-world networks into and across the local health and social care economy that we describe as CoPs. Consequently, this means that our implementation model reflects and responds to the multiple levels and actors that will need to be engaged and committed for the research to practice gap to be bridged and lead to the desired benefits for patients.

If the evident challenges can be overcome there may be many gains in terms of efficiency of conducting the research, and speed and breadth of translation of effective interventions into practice. We consider that the organisational learning approach to conducting and implementing research can be sustained and reach its full potential provided there is a supportive management structure and culture encompassing health and social care organisations and their academic partners, together with further coproduced research activity.

An organisational learning approach to knowledge mobilisation helps to make a service more amenable to constructive change and innovation through having a receptive implementation climate [61]. The organisation is prepared for the intervention, and importantly, the intervention fits the needs and values of the organisation, thus considerably enhancing its uptake of improvements to care. Consequently, we believe that our organisational learning approach can offer a lasting legacy to organisations that engage with it.

\section{Abbreviations}

ADHD: Attention deficit hyperactivity disorder; CAMHs: Child and adolescent mental health; CLAHRC: Collaboration for Leadership in Applied Health Research and Care; CoPs: Communities of practice; DF: Diffusion fellow; KTA: Knowledge to action; MRC: Medical Research Council;

NDL: Nottinghamshire, Derbyshire, Lincolnshire; NHS: National Health Service; NICE: National Institute for health and Clinical Effectiveness; NIHR: National Institute for Health Research; NRES: National Research Ethics Service; NPT: Normalisation process theory; PARIHS: Promoting action on research implementation in health services; PDSA: Plan-Do-Study-Act; POPULOS: Panel of people who use local services; QUERI: Quality enhancement research initiative; RCT: Randomised control trial.
}

\section{Competing interests}

The authors declare that they have no competing interests.

\section{Acknowledgements}

CLAHRC-NDL is funded by the National Institute for Health Research (NIHR) (2008-2013). Applicants for CLAHRC-NDL funding were Mike Cooke, Nick Manning, Christopher Hollis, Joe Kai, Marion Walker, Richard Morriss, Justine Schneider, Graeme Currie, Clive Adams, Anthony Avery, Anne Garland, Cris Glazebrook, Kevin Howells, Denise Kendrick, Mary McMurran, Nadeem Qureshi and Kapil Sayal. CLAHRC-NDL is a partnership between the University of Nottingham, NHS Nottingham City, NHS Nottinghamshire County, Nottingham University Hospitals NHS Trust, NHS Bassetlaw, NHS Derby City, NHS Derbyshire County, Derbyshire Healthcare Foundation Trust, Lincolnshire Partnership Foundation Trust, East Midlands Strategic Health Authority and Nottingham City and Nottinghamshire County local authorities. Ethical approval has been granted for all of the studies outlined in this paper (each study has a separate application). The views expressed in this publication are those of the authors and not necessarily those of our partner organisations, the NIHR or the Department of Health.

\section{Author details}

${ }^{1}$ CLAHRC-NDL/Nottingham University Business School, Institute of Mental Health, University of Nottingham Innovation Park Nottingham NG7 2TU, UK. ${ }^{2}$ CLAHRC-NDL/School of Community Health Sciences, Sir Colin Campbell Building, University of Nottingham Innovation Park, Nottingham NG7 2TU, UK. ${ }^{3}$ Warwick Business School, The University of Warwick, Coventry CV4 7AL, UK. ${ }^{4}$ CLAHRC-NDL/School of Sociology and Social Policy, Sir Colin Campbell Building, University of Nottingham Innovation Park, Nottingham NG7 2TU, UK.

\section{Authors' contributions}

The CLAHRC-NDL application to NIHR was developed by GC, RM and JS with the help of other applicants. ER led the writing of this paper. All authors have had the opportunity to share comments and make amendments. All of the authors have read and approved the final manuscript.

Received: 11 October 2011 Accepted: 3 May 2012 Published: 3 May 2012

\section{References}

1. Eccles M, Armstrong D, Baker R, Cleary K, Davies H, Davies S, Glasziou P, Illiot I, Kinmouth A-L, Leng G, Logan S, Marteau T, Michie S, Rogers H, RycroftMalone J, Sibbald B: An implementation research agenda. Implementation Sci 2009, 4:18.

2. Grol R, Grimshaw J: From best evidence to best practice: effective implementation of change in patients' care. Lancet 2003, 362:1225-1230.

3. Rycroft-Malone J, Wilkinson JE, Burton CR, Andrews G, Ariss S, Baker R, Dopson S, Graham I, Harvey G, Martin G, McCormack BG, Staniszewska S, Thompson C: Implementing health research through academic and clinical partnerships: a realistic evaluation of the Collaborations for Leadership in Applied Health Research and Care (CLAHRC). Implementation Sci 2011, 6:74.

4. Medical Research Council: A Framework for development and evaluation of RCTs for Complex Interventions to Improve Health. London: Medical Research Council; 2008.

5. Ward V, House A, Hamer S: Developing a framework for transferring knowledge into action: a thematic analysis of the literature. $J$ Health Serv Res Policy 2009, 14(3):156-164.

6. Dearing J: Applying diffusion of innovation theory to intervention development. Res Soc Work Prac 2009, 19(5):503-551.

7. Tooke J: Report of the High Level Group on Clinical Effectiveness, Chaired by Professor Sir John Tooke. London: Department of Health; 2007.

8. Cooksey D: A Review of UK Health Research Funding. London: The Stationary Office; 2006.

9. Smith R: What is 'implementation research' and what happened to GRIP? British Medical Journal group Blogs, [http://blogs.bmj.com/bmj/2011/02/01/ richard-smith-what-is-\%E2\%80\%9Cimplementation-research\%E2\%80\%9Dand-whatever-happened-to-grip/].

10. Kislov R, Harvey G, Walshe K: Collaborations for Leadership in Applied Health Research and Care: lessons from the theory of communities of practice. Implementation Sci 2011, 6:64.

11. Davies HTO, Nutley S, Walter I: Why 'knowledge transfer' is misconceived for applied social research. J Health Serv Res Policy 2008, 13:188-190.

12. Lomas J: The inbetween world of knowledge brokering. Br Med J 2007, 334:129-132. 
13. Nutley SM, Walter I, Davies HTO: Using Evidence: How research can inform public services. Bristol: Polity Press; 2007.

14. Crilly T, Jashapara A, Ferlie E: Research utilisation and knowledge mobilisation: a scoping review of the literature. London: Report for the National Institute for Health Research Delivery and Organisation Programme. HMSO; 2010.

15. Greenhalgh T, Robert G, Macfarlane F, Bate P, Kyriakidou O: Diffusion of Innovations in Service Organisations: Systematic Review and Recommendations. Milbank Q 2004, 82(4):581-629.

16. Eccles M, Grimshaw J, Walker A, Johnston M, Pitts N: Changing the behavior of healthcare professionals: the use of theory in promoting the uptake of research findings. J Clin Epidemiol 2005, 58:107-112.

17. Wilson KM, Brady TJ, Lesesne C, On behalf of the NCCDPHP Work Group on Translation: An organizing framework for translation in public health: the Knowledge to Action Framework. Prev Chronic Dis 2011, 8(2):A46.

18. Stetler CB, Damschroder LJ, Helfrich CD, Hagedorn HJ: A Guide for applying a revised version of the PARIHS framework for implementation. Implementation Sci 2011, 6(1):99.

19. May C: The clinical encounter and the problem of context. Socio/ 2007, 41:29-45.

20. May C: A rational model for assessing and evaluating complex interventions in health care. BMC Health Serv Res 2007, 6:86

21. May CR, Mair F, Finch T, MacFarlane A, Dowrick C, Treweek S, Rapley T, Ballini L, Ong BN, Rogers A, Murray E, Elwyn G, Légaré F, Gunn J, Montori VM: Development of a theory of implementation and integration: normalization process theory. Implementation Sci 2009, 21(4):29.

22. Rycroft-Malone J: The PARIHS framework-a framework for guiding the implementation of evidence-based practice. J Nurs Care Qual 2004, 19 (4):297-304.

23. NHS Institute for Innovation and Improvement. Quality and Service Improvement Tools: Plan-Do-Study-Act Cycle., [http://www.institute.nhs.uk/ quality and service improvement tools/ quality_and_service_improvement_tools/plan_do_study_act.html].

24. Grol R: Beliefs and evidence in changing clinical practice. British Medical Journal 1997, 315:418-421.

25. Currie G, Waring J, Finn R: The limits of knowledge management for public services modernisation: The case of patient safety and service quality. Public Adm 2008, 86(2):365-385.

26. Waring J, Currie G: Managing expert knowledge: organisational challenges and occupational futures for the UK medical profession. Organisation Stud 2009, 30(7):755-778.

27. Easterby-Smith M, Crossan M, Nicolini D: Organisational learning: debates past, present and future. J Manag Stud 2000, 37(6):783-796.

28. Ferlie E, Fitzgerald L, Wood C, Hawkins C: The nonspread of innovations: the mediating role of professionals. Acad Manage J 2005, 48(1):117-134.

29. Fitzgerald L, Ferlie E, Wood M, Hawkins C: Interlocking interactions, the diffusion of innovations in healthcare. Human Relations 2002, 55(12):1429-1449.

30. Graham ID, Logan J, Harrison MB, Strauss SE, Tetroe J, Caxwell W, Robinson $\mathrm{N}$ : Lost in knowledge translation: time for a map? J Contin Educ Health Prof 2006, 26:13-24.

31. Graham ID, Tetroe J: Some theoretical underpinnings of knowledge translation. Acad Emerg Med 2007, 14:936-941.

32. Graham ID, Tetroe J: How to translate health research knowledge into effective healthcare action. Healthc Q 2007, 10(3):20-22.

33. Lee RG, Garvin T: Moving from information transfer to information exchange in health and health care. Soc Sci Med 2003, 56:449-464

34. Rycroft-Malone J, Seers K, Tichen A, Harvey G, Kitson A, McCormack B: What counts as evidence in evidence-based practice? Adv J of Nurs 2004, 47(1):81-90

35. Gabbay J, le May A, Jefferson H, Webb D, Lovelock R, Powell J, Lathlean J: A cased study of knowledge management in multi-agency consumerinformed 'communities of practice': implications for evidence-based policy development in health and social care. Health 2003, 7(3):283-310.

36. Li L, Grimshaw J, Nielsen C, Judd M, Coyte P, Graham I: Use of communities of practice in business and healthcare sectors: A systematic review. Implementation Sci 2009, 4:27

37. Glasby J, Beresford P: Who knows best? Evidence-based practice and the service user contribution. Crit Soc Policy 2006, 26:268-284.

38. Ward PR, Thompson J, Barber R, Armitage CJ, Boote JD, Cooper CL, Jones GL: Critical perspectives on 'consumer involvement' in health research: epistemological dissonance and the know-do gap. J Socio/ 2010, 46(1):63-82.

39. Ferlie E, Shortell S: Improving the quality of health care in the United Kingdom and the United States: a framework for change. Milbank Q 2001, 79:281-315.
40. Easterby-Smith M, Lyles MA: Handbook of Organisational Learning and Knowledge Management. Oxford: Blackwell Publishing; 2005.

41. Suchman L: Plans and Situated Actions: the problem of human-machine communication. Cambridge: Cambridge University Press; 1987.

42. Brown JS, Duguid P: Knowledge and organisation: a social-practice perspective. Organisation Sci 2001, 12(2):198-213.

43. Lave J, Wenger E: Situated Learning: Legitimate Peripheral Participation. Cambridge: Cambridge University Press; 1991.

44. Wolff $\mathrm{N}$ : Using randomized controlled trials to evaluate socially complex services: problems, challenges and recommendations. J Ment Health Adm 2000, 3:97-109.

45. Mercer SL, DeVinney BJ, Fine LJ, Green LW, Dougherty D: Study designs for effectiveness and translation research: identifying trade-offs. Am J Prev Med 2007, 33:139-154.

46. Medical research council. Developing and evaluating complex interventions.

47. Denis JL, Langley A, Cazale L: Leadership and strategic change under ambiguity. Organisation Stud 1996, 17:673-699.

48. Rogers EM: Diffusion of Innovations. 5th edition. New York: Free Press; 2003.

49. Wenger $\mathrm{E}$ : Communities of practice and social learning systems. Organization 2000, 7:225

50. Thomson L, Schneider J, Wright N: Developing communities of practice to support the implementation of research into clinical practice. Leadersh Health Serv, in press.

51. NHS East Midlands: From Evidence to Excellence - our clinical vision for patient care. [http://www.excellence.eastmidlands.nhs.uk/welcome/].

52. Czarniawska B, Sevón G: Translating Organisational Change. Berlin: Walter de Gruyter; 1996.

53. Rowley E: Protocol for a qualitative study exploring the roles of 'Diffusion Fellows' in bridging the research to practice gap in the Nottinghamshire, Derbyshire and Lincolnshire Collaboration for Leadership in Applied Health Research and Care (CLAHRC-NDL). BMJ Open 2012, 2:e000604. doi:10.1136/bmjopen-2011-000604.

54. Morriss R, Marttunnen S, Garland A, Nixon N, McDonald R, Sweeney T, Flambert H, Fox R, Kaylor-Hughes C, James M, Yang M: Randomised controlled trial of the clinical and cost effectiveness of a specialist team for managing refractory unipolar depressive disorder. BMC Psychiatry 2010, 10:100.

55. Tsoukas H, Vladimirou E: What is organisation knowledge? J Manag Stud 2001, 38(7):973-993.

56. Schon DA: Beyond the Stable State. London: Norton and Company; 1971.

57. Schon DA: Technology and change: The new Heraclitus. New York: Delacorte Press; 1967.

58. Timmermans S, Berg M: The practice of medical technology. Sociol Health IIIn 2003, 25:97-114.

59. Oxman AD, Fretheim A, Flottorp S: Variance and dissent. The OFF theory of research utilization. J Clin Epidemiol 2005, 58:113-116.

60. Currie G, Suhomlinova O: The impact of institutional forces upon knowledge sharing in the UK NHS: the triumph of professional power and the inconsistency of policy. Public Adm 2006, 84(1):1-30.

61. Klein KJ, Sorra JS: The challenge of innovation implementation. Acad Manage Rev 1996, 21(4):1055-1080.

doi:10.1186/1748-5908-7-40

Cite this article as: Rowley et al:: Research into practice: Collaboration for Leadership in Applied Health Research and Care (CLAHRC) for Nottinghamshire, Derbyshire, Lincolnshire (NDL). Implementation Science 2012 7:40. 